\title{
Assistive Smart, Structured 3D Environmental Information for the Visually Impaired and Blind: Leveraging the INSPEX Concept
}

\author{
S. Lesecq, O. Debicki, L. Ouvry, \\ C. Fabre, N. Mareau, J. Foucault \\ CEA, LETI, Minatec Campus \\ F-38054 Grenoble Cedex, France \\ Email: suzanne.lesecq@cea.fr
}

\author{
S. Buckley, C. Jackson \\ SensL \\ Ireland
}

\author{
J. Barrett, A. McGibney, \\ S. Rea, D. Rojas \\ Cork Institute of Technology \\ Cork, Ireland
}

\author{
R. Banach, J. Razavi \\ University of Manchester \\ Manchester, U.K.
M. Correvon, G. Dudnik CSEM SA \\ 2002 Neuchatel, Switzerland \\ C. O'Murchu, A. Mathewson, R. O'Keeffe \\ Tyndall National Institute \\ Cork, Ireland
}

\author{
J.-M. Van Gyseghem, J. Herveg, N. Grandjean, F. Thiry \\ University of Namur \\ Namur, Belgium
}

\author{
A. di Matteo, V. Di Palma, F. Quaglia, G. Villa \\ STMicroelectronics Srl \\ 80022 Arzano, Naples, Italy
}

\begin{abstract}
Inspired by the abilities of contemporary autonomous vehicles to navigate with a high degree of effectiveness, the INSPEX Project ${ }^{1}$ seeks to minaturise the sensing and processing technology involved, to produce devices which can help navigate within an environment in a smart way, enabling their use in a wide variety of applications. The project is focused on producing an advanced prototype for a device which can be attached to a VIB person's white cane, and which, through the use of a variety of minaturised sensors, and of the processing of their data via sophisticated algorithms, can offer the user a richer palette of information about the environment than the use of the unadorned white cane alone could furnish. The various strands contributing to the project are overviewed, and the prospects for further enhancements are contemplated.
\end{abstract}

\section{INTRODUCTION}

Q IGNIFICANT visual impairment is highly disabling for $\checkmark$ any person since a huge proportion of the information people get from the world as a whole comes to them through the visual route. One of the most debilitating aspects of this is the impact on the ability of a visually impaired or blind (VIB) person to navigate unaided through the outdoor environment. Many blind people find themselves confined to their home, simply because venturing outside poses a challenge that they find too daunting.

One way of significantly alleviating this situation is to give the VIB person a guide dog. Guide dogs can be liberating for a VIB person to an almost miraculous degree [1]. A properly

\footnotetext{
${ }^{1}$ The INSPEX project has received funding from the European Union's Horizon 2020 research and innovation programme under grant agreement No 730953. The work was also supported in part by the Swiss Secretariat for Education, Research and Innovation (SERI) under Grant 16.0136 730953. We thank them for their support.
}

trained dog's intelligence can process the visual information it sees in the outside world to a level that compares well with that of a human - at least when it comes to recognising dangerous situations and seeing obstacles that should be avoided by its VIB handler. A good guide dog literally gives its VIB handler a new lease of life.

Unfortunately, good guide dogs are hard to come by demand far outstrips supply. A good guide dog must first be born of the right breed, and display a suitable temperament [2]. Then it must undergo extensive training as it grows from a puppy to an adult dog. Then it must be placed with a VIB owner who must be in a position to look after it, and the two of them must get along unproblematically [1]. None of this is easy, and it costs a lot per guide dog successfully placed.

Advancing technology offers some relief from this unhappy situation. The inspiration comes from autonomous vehicles [3], [4]. These days it is no novelty to see in the news stories of autonomous cars of various kinds navigating with apparent effectiveness through the streets of some city (but now, this can also have tragic consequences [5]). We are told that selfdriving cars (possessing a number of degrees of autonomy, graded from 0 (not autonomous at all) to 5 (fully autonomous and dependable in all situations)), are on the horizon. Such vehicles get their navigational capabilities by deploying a large number of sensors, and processing all the data that they produce via sophisticated algorithms whose goal is to produce a navigation strategy for the vehicle that human beings would regard as being approriate for the circumstances.

The growing familiarity of such a technological approach, combined with the increasing minaturisation of the needed hardware components opens the door to a much wider appli- 


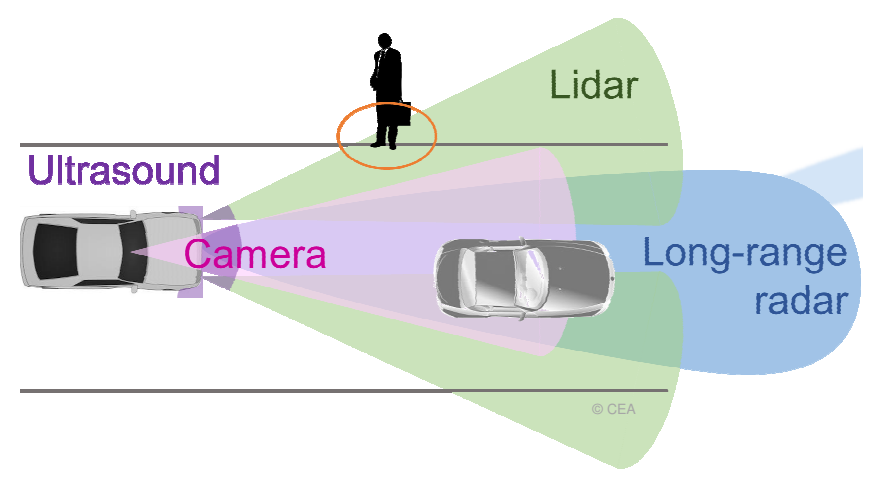

Fig. 1. Sensor deployment in an autonomous vehicle application.

cation of the underlying ideas. Autonomous vehicles have the luxury of being able to support an almost arbitrary collection of sensors and processors for this purpose, becasue of their default size, weight and on board power supply capabilities. Many other aplications, however, do not, but that does not make such applications any less desirable.

The INSPEX Project aims to produce an advanced prototype of a minaturised navigation aid that is at a wearable scale. The wearability requirement puts pressure on the number size, weight and power requirements that can be accommodated. While falling short of the full capabilities of an autonomous vehicle, the degree of minaturisation available within today's sensors puts a wearable device within reach. While such a device could be used (and in the longer term is fully intended to be used) for a variety of situations, the creation of an aid that will significantly assist VIB persons to navigate outdoors is the INSPEX Project's priority.

The rest of this paper is as follows. In Section II we briefly overview navigation in autonomous vehicles, and its implications. In Section III we look at how the INSPEX Project positions itself in the light of the preceding. Section IV focuses on the VIB use case. In Section V we outline the issues faced by the INSPEX design. Section VI looks at the INSPEX advanced prototype in detail, and Section VII describes the associated physical integration challenges. Section VIII looks at verification, validation and evaluation in INSPEX. Section IX surveys related systems and Section $\mathrm{X}$ concludes.

\section{NAVigation in Autonomous Vehicles}

The inspiration for the INSPEX Project comes from the growing capabilities of autonomous vehicles. Conceived in fiction over three dacades ago, autonomous vehicles started to become a serious reality within the last decade, especially the last five years. There are legion societal issues surrounding true vehicle autonomy, from attribution of liability when something really bad happens, to massive job losses due to a vanishing need for drivers. Our interest, though, is in the technology.

Fig. 1 shows a schematic of an autonomous vehicle system. The car on the road is equipped with LiDAR, with RADAR, with ultrasound (US) as well as a camera and vision system;

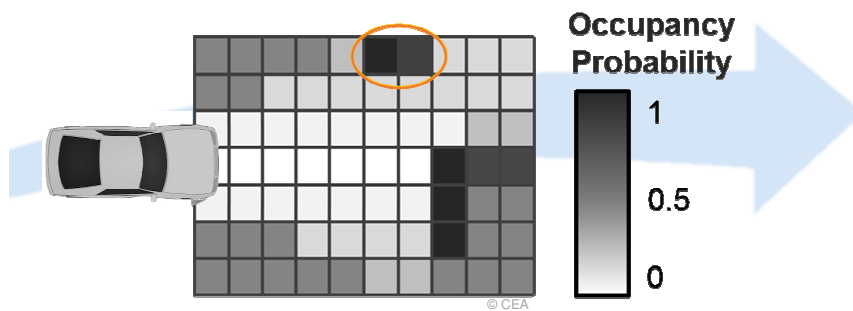

Fig. 2. Turning sensor data into an occupation grid.

not shown in the figure, there is also an IMU. On board the vehicle, there will be a GPS system together with a mapping application.

In a real driving situation, the data pouring from the sensors is gathered, timestamped, and then sent to a data fusion application that calculates, using Bayesian estimation, an occupancy grid [6], [7]. This divides the 3D space in front of the vehicle (and to the extent necessary, the sides and back too) into cubical sections, for each of which, an estimate is calculated of the probability of there being a obstacle of some sort present in that cube. Fig. 2 gives an idea of this, in which the car in front of the instrumented car of Fig. 1 is detected by the long range RADAR and the person standing at the side of the road is detected by the LiDAR sweeping to the side. The occupancy grid in Fig. 2 shows the higher probability of there being an obstacle in these positions by the denser shading in the relevant squares.

As noted earlier, the large size, weight and power of a vehicle permits a full suite of useful sensors to be deployed. In a minaturised scenario, this is no longer possible, and the main casualty is the vision system. It is not that cameras need to be large or heavy these days, but the processing required to make useful sense of a visual image would overwhelm the capacity of any battery light enough to be carried (and the capability of any computing equipment not connected to the cloud).

\section{The INSPEX PROJECT AND ITS USE CASES}

Taking on board that the contemporary trend in sensors of all kinds is towards ever smaller ever more low power versions of the basic capability of the sensor, INSPEX intercepts this trend to reappraise the autonomous vehicle obstacle detection and navigation architecture. The outcome of this is the concept of a small and lightweight device, minimising the resources needed for efficient occupation grid estimation [8], that offers the potential to enhance the way that navigational and positioning challenges are addressed in a wide variety of application areas.

Fig. 3 gives an indication of the range of applications whose working might benefit from the incorporation of INSPEX technology. The figure is divided into several sections. 


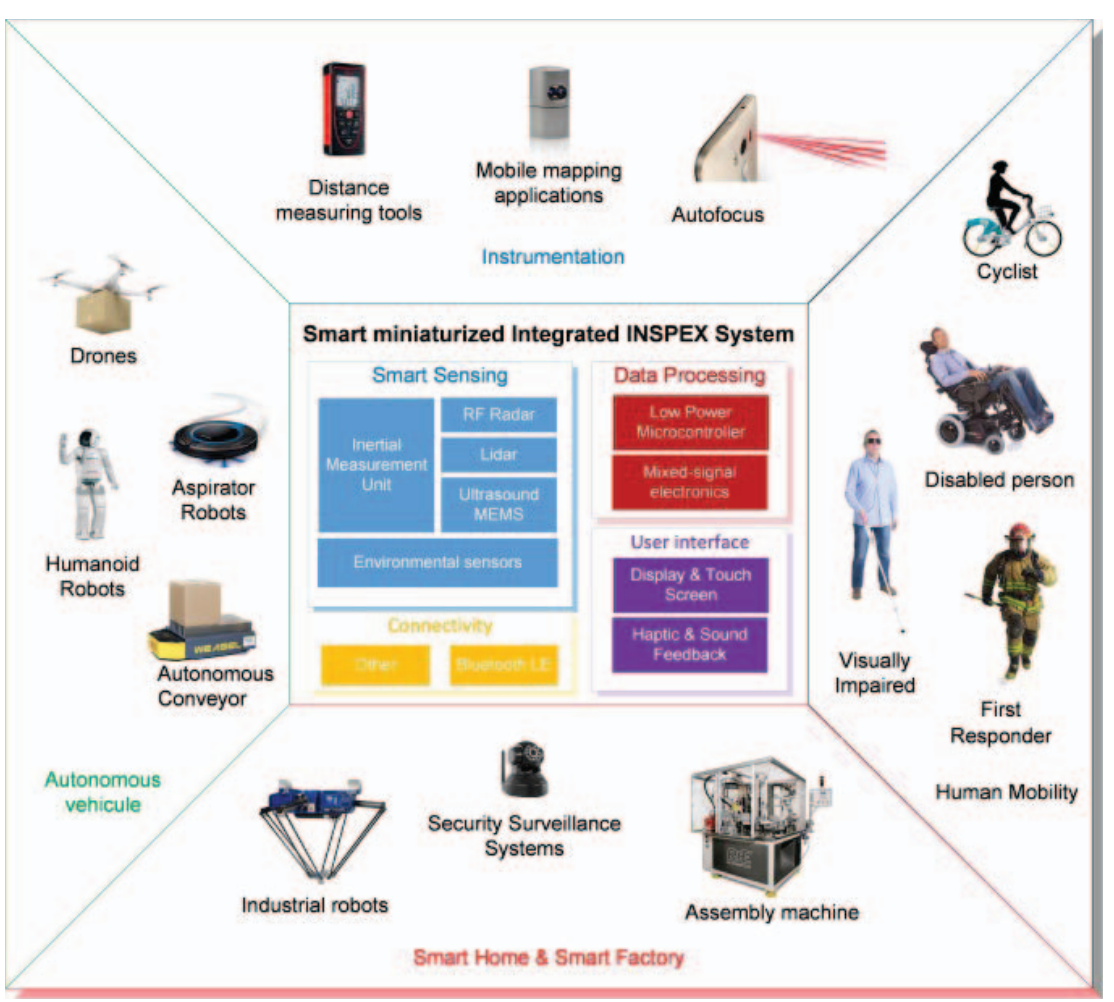

Fig. 3. A selection of potential INSPEX use cases.

On the right we see schematics for human centred applications. We see the VIB use case which forms the focus of the initial INSPEX development. Accompanying this are other use cases. The first responder use case covers situations like that of firefighters, who regularly need to attempt rescues and other complex retrieval operations in enclosed smoke filled (and thus opaque) environments and thus can benefit from non-visual cues about obstacles around them. The severely disabled who have obstacles to process the visual information from their surroundings can also benefit from INSPEX. There are also use cases for the non-disabled, such as cyclists and pedestrians. Such use cases arise, particularly in various highly poluted mega-cities, where, on a regular basis, smog levels reach such a pitch that visibility is seriously restricted.

At the top of Fig. 3 we have some use cases connected with instrumentation. Modern distance measuring tools typically make use of a single laser beam whose reflection is processed to come up with the numerical result. This strategy works well when the target is a hard, flat surface. For more uneven, and textured surfaces, the reading given (if any) may be subject to errors. An INSPEX enhancement to such instruments can yield better error detection and could perform better in a wider variety of situations. INSPEX could also improve to working of mobile mapping applications. Modern 3D environment capture instruments already use LiDARs to accurately measure the characteristics of their environment. Integrating more sensors into such instruments can improve their performance by better registering elements of the environment that are transparent to LiDAR. Similar remarks apply to autofocus applications.

These days, camera systems (typically in leading edge phones) employ increasingly sophisticated algorithms to distinguish foreground from background, to make up for varying lighting conditions, and generally to compensate for the user's lack of expertise in photography. Bringing in the augmented sensing capabilities of INSPEX opens the way to even more sophisticated approaches in this field.

At the bottom of Fig. 3 we have some use cases mainly concerned with navigation inside large, enclosed environments. Currently, highly automated factories can feature assembly lines consisting of hundreds of robots, each performing a specific task, within tightly constrained parameters. Such factories have to be planned and laid out with a great deal of precision because the industrial robots involved have limited intelligence. The proliferation of big data techniques generates an impetus to increase this robotic smartness as rapidly as possible, so that the detail needed in the planning phase can be reduced, and the robots involved can be more rapidly reconfigured to address the tasks spawned by the changing business environment. Adding INSPEX capabilities to the mix enhances the ability of the robots to accurately orient themselves relative to other significant elements of their working environment. And while the scenario just outlined focuses on fixed robots, the issues to be addressed become even more acute if the robots are mobile. Mobile robots need to accurately orient themselves within their environments, particularly so that they can avoid harm to humans who may be working in their vicinity. Also, security surveilance systems, which traditionally rely on infra-red sensors, can benefit from the enhanced precision that INSPEX can bring.

On the left of Fig. 3 we see some use cases in the autonomous small vehicle domain. When a vehicle is small, the effectively unrestricted tolerance of high weight, size and power consumption that large road vehicles can afford, disappears. Small airborne drones have demands of this kind that are every bit as exacting as those that we have in the VIB use case. As the use of drones increases, especially within cities, their need to accurately navigate within the urban environment rises significantly. Drones will increasingly need to manoeuvre within quite tightly constrained urban spaces, without colliding either with the fixed environment, or with each other. INSPEX capabilities can significantly address the sensing requirements of this use case. Humanoid robots have similar requirements since they are quite severely constrained regarding (especially) size and power consumption. INSPEX 
capabilities will increasingly become indispensable as such robots increasingly become autonomous and capable of independent action. Another use case highlighted in the figure is that of autonomous domestic robotic assistants. A typical floor cleaning robot will, these days, use a fairly hit and miss approach to finding parts of the floor to clean. Gradually, it learns where obstacles are, and builds an approximate map to improve its navigation subsequently. This works well of the furniture remains static. But if the user is inclined to move things around to repurpose the room in different ways, then the robot will remain in the early learning phase as its prior experience is frequently rendered unreliable. More accurate navigation capabilities acquired from equipment such as INSPEX can contribute to shortening the learning curve in these circumstances.

\section{The VIB Use Case Challenge}

The main focus of the INPSEX Project is the design and production of an advanced prototype obstacle detection and navigation system to assist visually impaired and blind users. According to the World Health Organisation, the number of VIB persons numbers 285 million and, due to an aging global population along with the debilitating health conditions that greater age brings, this number will double in twenty years or so [9]. The wide variety of conditions that a person may encounter in the outdoor environment makes the design of a system that gives really useful feedback to users in a convenient and informative way into a highly nontrivial challenge. The primary objective of INSPEX is to produce a clip-on device (together with the support facilities that it needs) that can add significant functionality to the principal navigational aid used by VIB persons, namely the white cane.

Obstacles to the VIB person's smooth and safe progress come in many forms. They include actual solid objects that impede the user - these may be found at many levels, from the ground, all the way up to head height. This variety of positions at which obstacles may be located poses a major problem for detection technologies, as an approach that detects at one position may be oblivious to obstacles elsewhere. Somehow, the whole range of the user's 3D space must be monitored and the user suitably informed.

Obstacles on the ground are dealt with quite effectively using the white cane. The experienced VIB user can gain a lot of information, especially along familiar routes, from the minor variations in the surface texture that they sense with their white cane. The specially textured tiling that has been introduced along urban pavements, particularly at junctions, and along the edges of pavements and platforms for light and heavy rail, are a deliberate attempt to increase the informativeness of the surface for the disabled user, and for the VIB community in particular [10].

In urban environments, especially in changeable ones, collisions with obstacles at chest or head height are a significant risk factor for the VIB community. VIB persons may wear headgear, even if for sartorial reasons they might prefer not to, to offer at least some cushioning in case of unanticipated collisions. Such strategies offer, at best, only partial defense against the risk, and injuries sustained by VIB persons are a de facto unavoidable feature of a VIB individual's efforts to participate in normal life [11]. In theory, VIB persons could choose to wear crash helmets and similar gear, but such items are felt to be extremely sigmatising, and so are eschewed by the VIB community.

We have been speaking about solid obstacles with which VIB persons might collide, but these are not the only hazards to worry about. Danger for the VIB person arises from approaching empty holes in the surface and holes filled with water (or other materials, whether liquid or not). Staircases comprise a vital element of the contemporary urban landscape, and constitute a particular risk factor for the VIB, especially when the staircase is a descending set. (It is worth observing that escalators, while being similar to stairs, are less risky for VIB persons, since they emit a typical 'escalator rumble' which warns the VIB person of their proximity.)

Thus a wide variety of sensing capabilities is needed to address all of these situations. Moreover, different meteorological conditions can significantly affect what a sensing instrument can acquire. Each different kind of precipitation has its own transparency/opacity characteristics, impacting the fidelity of the information that can be inferred from different sensors under different conditions.

\section{INSPEX VIB DESIGN ISSUES}

It is very tempting, when developing a technology to meet a user need, for the technologists to get completely absorbed in the fine details of the technical aspects and to drift away from involvement in the needs of the prospective user community. This simply decreases the degree to which the end result actually meets the original need.

To forestall this eventuality in INSPEX, an early phase of the design process was focused on user needs. Significant effort was expended to engage with associations for the VIB community to elicit the requirements that improved mobility aids ought to address. Following this engagement, a collection of personas were constructed in order that typical activities for such personas could be identified, to ensure that the eventual prototype that would be developed could be validated against these identified activities.

With this preparatory foundation addressed, the design phase could proceed in earnest. The overall architecture of the INSPEX system consists of three modules. There is the hardware module that attaches to the VIB person's white cane. This module communicates via Bluetooth with a software component running on a smartphone belonging to the user. The software component is able to use the phone's capabilities to communicate with the outside world, and to integrate information received from there with informaiton gained locally, in order to give the VIB user a richer and more informative experience. Finally, there is a pair of extraauricular headphones, connected to the smartphone module again via Bluetooth, that is able to paint a sound picture for the VIB user about their environment that is intended to be as vivid 


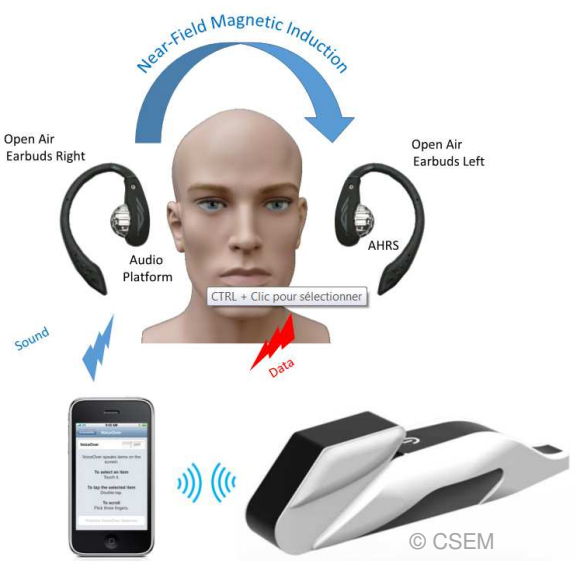

Fig. 4. The complete INSPEX system for the VIB use case.

and informative as possible. Fig. 4 shows these components in digramatic form.

The account just given makes it clear that INSPEX belongs very much in the Internet of Things family of systems. An INSPEX system is ready to receive information made available by the environment, in order to assist its user to gain a more accurate picture of where, in relation to the rest of the environment, he or she might be. This potentially raises various ethical concerns. These days many smart apps are designed to divulge data about their users, whether to the app manufacturer or to connected nodes in the wider environment, and it is the users' (assumed informed) choice about whether or not they are prepared to have such information divulged. By contrast, from a legal perspective, the VIB use case of INSPEX is generally regarded as a Class 1 medical device [12], [13]. Because of the medical connection, by default, a much larger quantity of regulation comes to bear on the device (although the regulatory situation may change in future). Moreover, the details of the regulations concerned vary from jurisdiction to jurisdiction, so ensuring that INSPEX conforms to all required regulation forms a significant strand of the project (handled by partner Univ. Namur). By default, INSPEX implements a privacy by design policy. No data (that is intelligible outside the INSPEX system) is ever made available to the environment.

Fig. 5 illustrates a prototype configuration of a VIB user deploying a white cane augmented with the kind of sensing abilities envisaged for the INSPEX white cane hardware clipon module. In fact the figure shows an early conceptualisation of the clip-on module, when having an additional module near the bottom of the white cane, to more precisely monitor conditions near the ground, was contemplated. In actual fact, consideration of the user feedback gained from the community and persona studies earlier in the process, quickly convinced the project consortium that the leverage around the

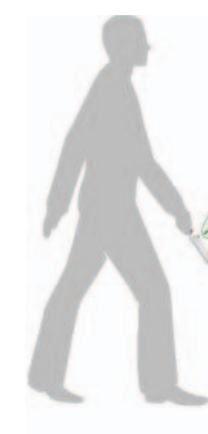

white cane's point of support from even a very light component attached near the end of the white cane would rapidly generate an unacceptable strain on the user's wrist (not to mention the added complexity, from the user's perspective, of having to deal with one more device, most likely with its own charging regime), rendering such a design unacceptable, even if it led to some engineering advantages. Therefore, the current design of the hardware clip-on module is a single unit attached near the point of support of the white cane by the user.

\section{The INSPEX AdVANCEd PRototype System}

In the light of the many and varied environmental circumstances that a VIB person may meet outdoors, the hardware clip-on module features a collection of sensors, that are intended to harvest the maximum amount of useful data in the widest possible range of conditions, bearing in mind the limitations of size, weight and power consumption. In this section, we overview the various components that comprise the INSPEX advanced prototype.

The heart of the INSPEX sensing system consists of four main sensors. There is a short range LIDAR, a long range LIDAR, a MEMS ultrasound sensor and a ultrawideband RADAR. These are supplemented with an inertial measurement unit and appropriate computation and communication facilities. The collection of sensors that have been incorporated into INSPEX complement each other in important ways, making up for each others' deficiencies under different circumstances in ways that genuinely add value [14].

For example, ultrasound sensors have a limited range (typically not exceeding $3 \mathrm{~m}$ ). While a $3 \mathrm{~m}$ range is largely adequate for the VIB use case, it is insufficient for many other use cases identified earlier, such as drones, autofocus, and surveilance systems.

Sensors based on lasers can see much further, but have other deficiencies, such as their relatively poor performance under conditions in which the ambient light shares frequencies or harmonics with the sensing system. They also perform poorly in detecting objects that are transparent to their frequencies; likewise highly reflective surfaces which do not scatter widely enough to send enough energy back along the incident beam.

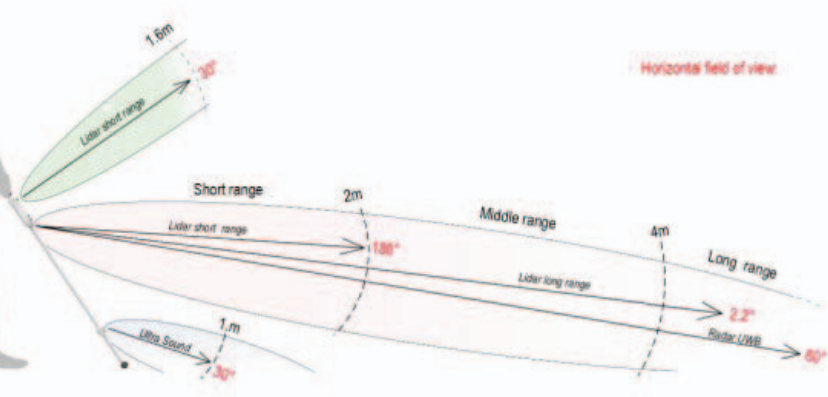

Fig. 5. Prototype INSPEX VIB white cane use case. 


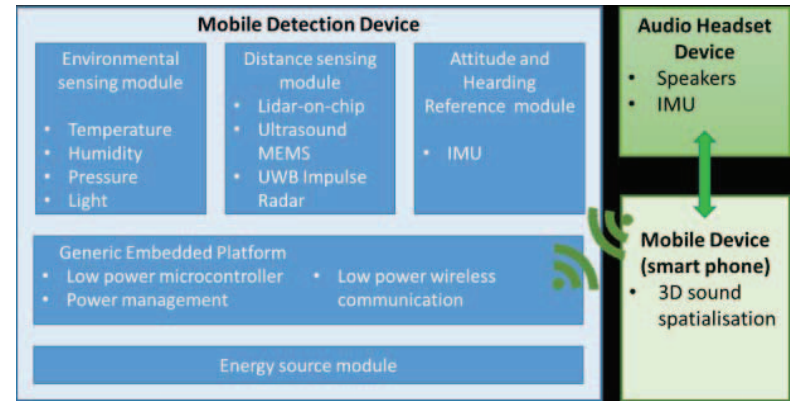

Fig. 6. The architecture of the INSPEX system.

Radio frequency radar, which utilises a different part of the electromagnetic spectrum than lasers, can perform much better when lasers are impeded by poor weather conditions such as rain, sleet, snow, hail. RF radar is also unimpeded by small obstacles, due to its wavelength. These response characteristics are also very different from the mechanical response of obstacles to ultrasound. It is thus clear that three technologies mentioned do offer the good complementarity claimed.

\section{A. Overall INSPEX Architecture}

Fig. 6 shows all the components of INSPEX. In the main Mobile Detection Device we see not only the distance sensing module whose elements we have just discussed, but also other modules. There is an Environmental Sensing Module. This provides information about the ambient circumstances of use of INSPEX to improve the decision making capability of the overall system about how to best configure itself for optimal performance. Vital to effective working is the Attitude and Heading Reference Module. This contains an Inertial Measurement Unit, which regularly feeds information about the physical orientation in real space of the whole Mobile Detection Device to the system's decision making capability. This is indispensable as all the distance measurements obtained by the distance sensing devices, must be correlated to the direction in $3 \mathrm{D}$ space in which they were made, in order for them to be useful in constructing a 3D occupancy grid. Energy is supplied to the whole Mobile Detection Device by the Energy Source Module, which is an interchangeable battery pack that can be quickly swapped for a spare when power is low, and which offers rapid recharge characteristics. The Generic Embedded Platform coordinates and controls the activities of the other modules in the Mobile Detection Device. As well as this it takes care of communication with what is referred to as the Mobile Device (Smart Phone) in Fig. 6.

A separate major component is the Audio Headset. This features a pair of extra-auricular earbuds to project the audio signal from INSPEX into the user's ears. It also contains another Inertial Measurement Unit, so that the orientation of the user's head may be inferred, in order that the calculated INSPEX audio signal is perceived to be oriented stably with respect to a 3D space inertial frame.
The final major component of the system is the smartphone. This is connected to both the Mobile Detection Device and the Audio Headset via Bluetooth. It handles the IoT element of INSPEX's functionality which listens out for IoT beacons in the environment, information which is integrated into the audio signal presented to the user. It also correlates the 3D information of both Mobile Detection Device and Audio Headset to ensure the $3 \mathrm{D}$ space inertial frame stability required by the user.

\section{B. The INSPEX Development Trajectory}

The INSPEX development trajectory forsees the achievement of the final advanced prototype through a series of milestones, one per year, each producing a working version of the system that partly realises the project goals. Thus, the first year of the project produced $\mathrm{P} 0$, the first (non-minaturised) prototype. The second year produces $\mathrm{P} 1$, featuring the first stage of minaturisation and integration. The third year culminates in $\mathrm{P} 2$, the final prototype, in which minaturisation and integration reach their target level.

In the subsections that follow, we examine the various elements of INSPEX that have been introduced earlier in a little more detail. We start with the various sensors, and we illustrate the description of these with pictures of their early P0 prototypes. These were the only ones that had been manufactured and shown to be working at the time of writing of this paper.

\section{The Ultrawideband RADAR (CEA)}

The INSPEX RADAR sensor, developed by partner CEA, is an ultrawideband radar designed to detect movement in obstacles located up to $20 \mathrm{~m}$ away. Refreshing at up to $100 \mathrm{~Hz}$ will make it capable of high performance tracking of moving targets. This is facilitated by an aperture of $60^{\circ}$, giving the radar a reasonably wide viewing angle.

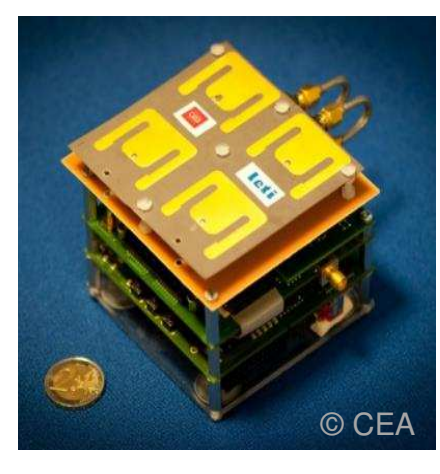

Fig. 7. The UWB Radar (CEA).
Fig. 7 shows an early pro-

totype of the radar. Minaturisation of such radars is not readily seen in the electronic component market, limiting the prospects for INSPEX. As INSPEX develops towards its final version, the first prototype will be minaturised to the extent that is feasible, with the circuit boards visible in the figure reduced in size. The antenna elements allow little scope for size or weight reduction.

\section{The MEMS Ultrasound (ST Microelectronics)}

The INSPEX ultrasound sensor, developed by partner ST Microelectronics, is a microelectromechanical system, or MEMS sensor. Of the two types of these that are available, 
capacitance based and piezoelectric effect based, the piezoelectric kind offers much lower power requirements, so is preferred for use in INSPEX. Fig. 8 shows an early prototype implementation for INSPEX. The size of the circuit board will decrease significantly in the later stages of the INSPEX project.

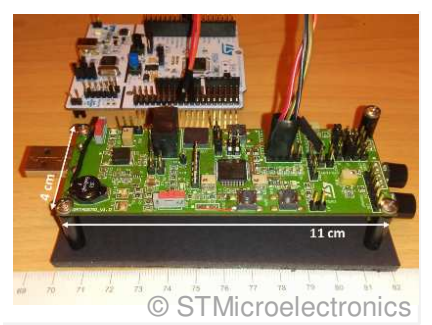

Fig. 8. The MEMS ultrasound sensor (ST Microelectronics).

in a large proportion of modern cars.

Ultrasound sensors have been used in diverse applications for some time now, for example, nondestructive testing, flow metering and medical imaging. The application perhaps most familiar to the man in the street is the collision warning system, to be found in various guises, such as parking assistance systems,

\section{E. The Long Range LiDAR (Tyndall, SensL)}

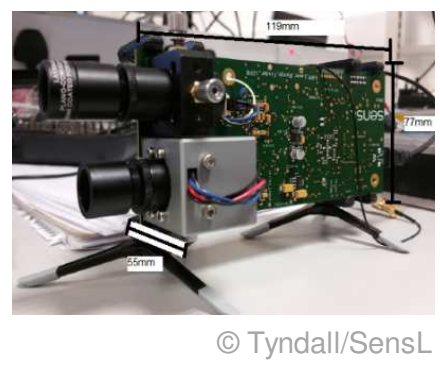

The Long Range LiDAR sensor for INSPEX, developed by project partners Tyndall and SensL, is a narrow beam $\left(2.2^{\circ}\right)$ LiDAR with a range of around $20 \mathrm{~m}$ or more. An early prototype appears in Fig. 9. The long range is useful for recognising distant hazards, however the narrow beam angle militates against utility. To mitigate this disadvantage, Fig. 9. The long range LiDAR (Tyndall, SensL)

an array version, utilising 64 channels is being developed for INSPEX. This will effectively scan (a portion of) a plane passing through the sensor, registering the distance away of obstacles encountered by the beams that radiate from the sensor in directions embedded in the plane.

Given the natural orientation of the Mobile Detection Device on the user's white cane, the plane will be oriented vertically, and so the user will gain a picture of obstacles at larger distances by sweeping the white cane from side to side, in a motion that departs little from a VIB person's natural use of a non-INSPEX-enhanced white cane. Further development of the sensor will be principally focused on reducing the size of the circuit board.

\section{F. The Short Range LiDAR (CSEM, CEA)}

The Short Range LiDAR sensor for INSPEX, is developed by project partners CSEM and CEA. It is based on an offthe-shelf component which is shown in Fig. 10. This LiDAR component has a larger field of view (around $30^{\circ}$ ) and a range of around $2 \mathrm{~m}$.

As in the long range $\mathrm{Li}$ DAR case, the basic OTS component will be integrated into a second generation sensor capable of looking at 9 independent measurement points within its field of view. Also as for the long range LiDAR case, development effort for the short range case will minaturise the electronics, leading to a much smaller circuit board in the final prototype.

Fig. 11 shows an early

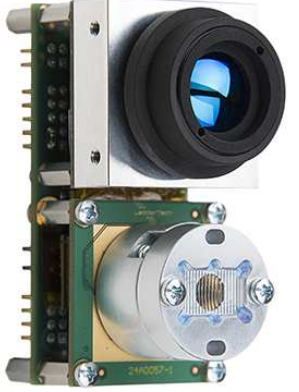

(C) LeddarTech

Fig. 10. The short range LiDAR COTS element.

version of the short range LiDAR integrated into a configuration that can sweep a plane, in the manner described for the long range LiDAR case.

\section{G. The IMU}

The Inertial Measurement Unit in the Mobile Detection Device of INSPEX is an off-the-shelf component, these devices being small enough these days to use without adaptation (this, in turn, being due to their mass use in mobile phones). Its job is to regularly supply information about its orientation and displacement in Euclidean space (and thus about the orientation and

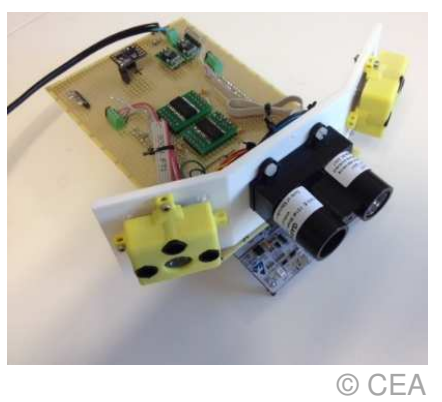

Fig. 11. The short range LiDAR in wide angle configuration (CEA CSEM). displacement of the Mobile Detection Device itself, and therefore about the orientation and displacement of the sensors contained within it) to the Generic Embedded Platform. This is needed so that the distance information coming from the other sensors can be correctly related to the current state and orientation of the occupation grid.

\section{H. Software and Firmware in the Generic Embedded Platform}

The Generic Embedded Platform within the INSPEX Mobile Detection Device has the responsibility of appropriately integrating all the sources of information provided by the family of sensors embedded in the Mobile Detection Device, and communicating suitably with the INSPEX Smartphone Application. Considering the variety of the information received, the integration of all this information is a non-trivial task, and a considerable quantity of sophisticated software is needed to accomplish an optimal result.

A major portion of the job is the receipt and husbanding of the raw data coming from the sensors. These all work at their own rate, modulated by the commands sent to them by the GEP. The data has to be received and timestamped so that fresh data may be given priority over older stale data. The raw 
distance data has also to be correlated with data from the IMU so that the orientation in space of the distance measurement may be discerned.

Once the raw data has been correctly marshaled, it is passed to the occupation grid computation, a proprietary component supplied by partner CEA. The CEA occupation grid computation is distinguished by its significantly greater efficiency [8], compared with standard Bayesian estimation algorithms, with their reliance on heavy duty floating point calculation[6], [7], which has a significant impact on power consumption in a portable lightweight system such as INSPEX.

Once the occupation grid has been derived, it is transmitted via Bluetooth to the INSPEX smartphone application. The granularity of the occupation grid is heavily constrained by the bandwidth available for this communication, and this places a fundamental limit on the detail about the environment that can be communicated to the user (although, see the discussion about this in Section VI-K).

\section{Power Management in the Mobile Detection Device}

The Mobile Detection Device contains a significant number of hardware components. Albeit that these are all ultimately minaturised low power components, they all still require power. As invariably happens in projects of this kind, each component in fact consumes more power than initial estimates stated, so significant effort has to be invested to reduce this, and to deploy every means possible to optimise power use. ${ }^{2}$

In INSPEX, a sophisticated power management strategy is utilised to eke out the energy made available by the energy source module to the greatest effect. A formal modelling approach is used to organise the various factors that have an impact on the consumption of energy into a consistent approach, consisting of three layers.

At the simplest level there is the correctness layer, which ensures that no hardware element is left running when it should not be doing so, and handles similar concerns. Next is the quantitative layer, which enhances the preceding with quantitative information, enabling estimates to be derived about power consumption and system uptime under different circumstances. Lastly comes the policy layer, which imposes constraints that take environmental and other considerations into account, enabling best use of remaining power according to higher level, user determined goals.

\section{J. The Audio Headset}

The INSPEX audio headset performs two important roles within the functioning of the whole system. It contains a pair of extra-auricular earbuds whose job it is to project the audio signal received via Bluetooth from the INSPEX smartphone application into the user's ears. The earbuds are extra-auricular in order that the user not be prevented from hearing sound from the environment itself. Many VIB persons report that they can 'hear' all sorts of elements within their environment (e.g. doors open or closed within the indoor environment, or

\footnotetext{
${ }^{2}$ Exactly the same remarks invariably apply to the overall weight of the integrated system.
}

walls and lampposts within the outdoor environment) unaided, so it is vitally important that they should not be deprived of this ability when using INSPEX, or else the takeup of the INSPEX VIB system by its target market will be very poor.

The INSPEX audio headset also contains another IMU. Thus the second job done by the audio headset is to send regular information about the orientation and displacement in Euclidean space of the audio headset to the INSPEX smartphone application. This enables the binaural audio signal received from the smartphone to consist of sounds that are be computed to have a stable point of origin in Euclidean space, despite the movement of the user's head.

\section{K. The INSPEX Smartphone Application}

The INSPEX Smartphone Application, developed by partner CIT, binds the functionality of the INSPEX modules discussed above into a consistent whole. One major task is the integrated handling of all the 3D information coming from different parts of the system. The mobile detection device is physically independent from the audio headset, so each is independently situated in Euclidean space. The information coming from the two IMUs in these modules has to be integrated so that a single consistent picture of the INSPEX system's position in 3D space can be determined. The calculations for this, though not trivial, are relatively familiar from the robotics world [15], [16].

Another major responsibility of the smartphone application is context awareness. In the growing world of IoT, especially as realised in the growing number of smart cities, the environment is capable of being, of itself, informative in the cyberspace domain. A smartphone will be capable of picking up this information from smart beacons as its user moves through such a smart city. Although a significant proportion of the smart beacons implemented in smart cities will have a commercial dimension, a proportion will be intended to act as aids to disabled users, especially the VIB community. The INSPEX smartphone application will be able to receive the signals from these, and to integrate it into the audio information transmitted to the user.

A final major responsibility of the smartphone application will be synthesising the sounds to be transmitted to the audio headset via Bluetooth. Here there is a significant challenge. The visual domain is not only very rich due to the high resolution available in visible frequencies, but is also three dimensional. By contrast, the audible domain, although also having a spectral range, is much more compressed than the visible spectrum, and sound needs a temporal element to be heard, whereas a visual impression can be gained almost instantaneously. How then to design audio feedback to users that accommodates the following conflicting requirements:

- the varying capability to overlay audio information onto ambient sound from the environment according to the ambient sound level and characteristics,

- the user's desire to hear the natural environment clearly,

- the user's desire not to be overloaded with audio information that is too complicated to absorb, 
- the user's desire to receive rich audio information, especially about the most important hazards in the environment, whatever they might be.

INSPEX partner GoSense is responsible for this constituent of the design, which is tackled via an Augmented Reality Audio approach. While good progress is anticipated with this approach, one can imagine an ongoing artificial intelligence challenge, stretching beyond the project's lifetime, to discover how to best tailor the audio feedback, in the face of the conflicting requirements listed, for different types of users, who can have very different preferences regarding what works best for them.

\section{PHYSICAL INTEGRATION}

One of the major targets of INSPEX is systems integration to achieve a small volume $\left(100 \mathrm{~cm}^{3}\right)$, lightweight $(200 \mathrm{~g})$ system 'Box' that meets the requirements of the VIB application. Into this Box must be integrated all of the obstacle detection transducers along with their signal processing boards, the main processing board, battery, power management and all cabling, connectors and switches.

Beyond the above, there are many other physical and practical constraints to be taken into account. Thus, each obstacle detection transducer must be orientated to point in the appropriate direction, and EMC issues must be managed properly inside the densely-packed Box. The Box and its contents must be robust against the mechanical impacts and vibrations induced by cane use and impact with obstacles, and the Box must be weather- and dust-proof while still allowing each obstacle detection transducer to transmit and receive through the Box wall. It must be possible for the VIB user to easily attach and detach the Box from the white cane either for off-cane charging or to allow the cane to be used without the INSPEX System if the user is, for instance, using the cane in a very familiar area. And to minimise imbalance and strains that could lead to user fatigue, the Box also be weight-balanced on the cane, with its centre of gravity as close as possible to the long axis of the cane. Finally, the Box should be aesthetically pleasing and easy to clean for the user.

Combined, these requirements represent a major hardware design challenge that must be addressed at every level of the system, combining both bottom-up and top-down design approaches, and many variations on systems integrations concepts, such as shown in Figure 12, to iterate to a wholesystem solution. Partner CIT takes care of these aspects of the INSPEX development.

\section{VERIFICATION, VALIDATION, EVALUATION}

A system as complex as our presentation of INSPEX has elucidated, evidently throws up highly non-trivial issues regarding the ensuring of correct behaviour under all operating conditions. This challenge must be approached in a disciplined and structured way, otherwise little assurance is derived.

At the lowest level, individual project partners are responsible for the testing and verification of their individual submodules. Usual best practice in each individual physical

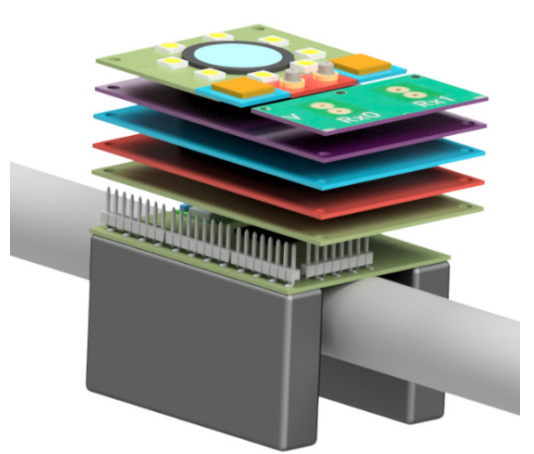

Fig. 12. Prototype INSPEX VIB physical configuration.

domain is applied in each case. At the next level up, the behaviour of the community of sensors must be integrated via the software and firmware of the generic embedded platform. Here, software complexity must be confronted. In this sphere, formal modelling and verification techniques are brought to bear. Since so much of the system depends on the behaviour of physical systems, which, compared with software systems, are considerably less negotiable (in the sense that one might simply change one's mind about what software should do —almost at will— whereas with physical devices, one is restricted to what the laws of nature allow and device engineering will permit), the basic objectives and low level aspects must come from the hardware development. Once those aspects are clear however, the higher level aspects can be modelled using a formal framework such as Event-B [17], [18]. This done, the required consistency of the models can be established, and refinement techniques can progress the models close to code. Partner Univ. Manchester is responsible for this interworking between formal and conventional development techniques. The result of all the activity that has been described can then be confronted with the project verification plan, which was drawn up a priori to define correct operation for INSPEX.

Once correct operation has been established, validation against user requirements can be done, to yield an evaluation of the achievements of INSPEX against real user needs. A number of VIB user communities are in communication with the project team, and will contribute to such user evaluations after the technical development has been completed.

\section{Related Systems}

The idea of creating an assistive device to help VIB persons to navigate safely, that functioned by translating information gained via wave physics based sensing into aural or tactile information communicable to a VIB person, does not originate with INSPEX. Among existing systems we can mention Smartcane [19], Ultracane [20], Bawa [21], Rango [22], etc.

Aside from these initiatives that are focused on making white canes smart, there are many devices that are deployed via mechanisms such as headwear of one kind or another, clip-on devices that clip on to the user's belt, or devices that can be strapped on to the chest or hung round the neck. 
However, the reaction from the VIB community to these proposals has been largely negative, as they are perceived to be stigmatising to a significant degree. And besides these products that primarily originate in the SME sector, the increasing promotion of societal assistance for citizens that suffer from various disabilities spurs an increasing interest from large corporations, e.g. [23].

The key observation about earlier systems is that they typically use a single sensing channel to probe the $3 \mathrm{D}$ environment in front of the user. This reduces the task of translating the information obtained into a format comprehensible by the user to the mere indication of a single distance measurement (in the simplest cases). This simplification avoids the complexities and costs of data fusion, at the price of forcing the toleration of any weak elements or 'blind spots' that are inherent in the physics of the given sensing channel. INSPEX is one of the eariest VIB assistive devices that is intrinsically multi-channel, although it is seen that more and more such devices are being proposed nowadays.

\section{CONClusion}

In the preceding sections we have introduced the INSPEX Project, and its ambitious retinue of imaginative use cases. The project currently focuses on the VIB use case, and the issues raised by this use case have been elaborated in detail in this paper. When the advanced prototype for the smart enhancement to the white cane has been completed, development will be pursued further towards a fully fledged commercial product. This subsequent phase will seek to further reduce the size, power consumption and weight of the unit, and will endeavour to locate a price point that will render it attractive to the VIB community. It is anticipated that the fruitful exploitation of this INSPEX use case will act as a spur to the devlopment of further commercially exploited use cases from among the wide selection described in Section III.

\section{REFERENCES}

[1] L. Whitmarsh, "The Benefits of Guide Dog Ownership," Visual Impairment Research, vol. 7, pp. 27-42, 2005.
[2] E. Wilsson and P.-E. Sundgren, "The Use of a Behaviour Test for the Selection of Dogs for Service and Breeding, I and II," Applied Animal Behaviour Science, vol. 53 and 54, pp. 279-295 and 235-241, 1997.

[3] Z. Qu, Cooperative Control of Dynamical Systems: Applications to Autonomous Vehicles. Springer, 2009.

[4] M. Fausten, "Evolution or Revolution: Architecture of AD Cars," in Proc. IEEE ESWEEK, 2015.

[5] UBER Self Driving Car Fatality, https://www.theguardian.com/technology/ 2018/mar/19/uber-self-driving-car-kills-woman-arizona-tempe.

[6] S. Thrun, W. Burgard, and D. Fox, Probabilistic Robotics. MIT Press, 2005.

[7] Moravec, H. and Elfes, A., "High Resolution Maps from Wide Angle Sonar," in Proc. IEEE ICRA, 1985.

[8] Dia, R. and Mottin, J. and Rakotavao, T. and Puschini, D. and Lesecq, S., "Evaluation of Occupancy Grid Resolution through a Novel Approach for Inverse Sensor Modeling," in Proc. IFAC World Congress, FACPapersOnLine, vol. 50, 2017, pp. 13 841-13847.

[9] WHO. Visual Impairment and Blindness, http://www.who.int/mediacentre/ factsheets/fs282/en/.

[10] T. Rosburg, "Tactile Ground Surface Indicators in Public Places," in Human Haptic Perception: Basics and Applications, Grunwald, Ed.

Springer, Birkhauser, 2008.
[11] R. Mandruchi and S. Kurniavan, "Mobility-Related Accidents Experienced by People with Visual Impairment," Insight: Research and Practice in Visual Impairment and Blindness, 2011.

[12] V. Theisz, Medical Device Regulatory Practices: An International Perspective. Pan Stanford Publishing, 2015.

[13] Wikipedia: Medical Devices, https://en.wikipedia.org/wiki/Medical_device.

[14] L. Scalise, V. Primiani, and P. Russo, "Experimental Investigation of Electromagnetic Obstacle Detection for Visually Impaired Users: A Comparison with Ultrasonic Sensing," IEEE Trans. on Inst. and Meas., vol. 61, pp. 3047-3057, 2012.

[15] R. Paul, Robot Manipulators: Mathematics, Programming, and Control. MIT Press, 1982.

[16] R. Murray, Z. Li, and S. Sastry, A Mathematical Introduction to Robotic Manipulation. CRC Press, 1994.

[17] J.-R. Abrial, Modeling in Event-B: System and Software Engineering. CUP, 2010.

[18] RODIN Tool, http://www.event-b.org/ http://sourceforge.net/projects/rodinb-sharp/.

[19] Smartcane, https://www.phoenixmedicalsystems.com/assistive-technology/ smartcane/.

[20] Ultracane, https://www.ultracane.com/.

[21] Bawa, https://www.bawa.tech/.

[22] Rango, http://www.gosense.com/rango/.

[23] Apple VIB Navigation Announcement, https://appleinsider.com/articles/ 18/06/28/apple-mulls-system-for-helping-visually-impaired-navigate-environ ment. 